\title{
The Existence of Fixed Points for Nonlinear Contractive Maps in Metric Spaces with $w$-Distances
}

\author{
Hossein Lakzian' ${ }^{1}$ and Ing-Jer Lin $^{2}$ \\ ${ }^{1}$ Department of Mathematics, Payame Noor University, Tehran 19395-4697, Iran \\ ${ }^{2}$ Department of Mathematics, National Kaohsiung Normal University, Kaohsiung 824, Taiwan \\ Correspondence should be addressed to Ing-Jer Lin, ijlin@nknu.edu.tw
}

Received 20 November 2011; Accepted 4 December 2011

Academic Editor: Yeong-Cheng Liou

Copyright (c) 2012 H. Lakzian and I.-J. Lin. This is an open access article distributed under the Creative Commons Attribution License, which permits unrestricted use, distribution, and reproduction in any medium, provided the original work is properly cited.

Some fixed point theorems for $(\varphi, \psi, p)$-contractive maps and $(\varphi, k, p)$-contractive maps on a complete metric space are proved. Presented fixed point theorems generalize many results existing in the literature.

\section{Introduction and Preliminaries}

Branciari [1] established a fixed point result for an integral type inequality, which is a generalization of Banach contraction principle. Kada et al. [2] introduced and studied the concept of $w$-distance on a metric space. They give examples of $w$-distances and improved Caristi's fixed point theorem, Ekeland's $\epsilon$-variational's principle, and the nonconvex minimization theorem according to Takahashi (see many useful examples and results on wdistance in [2-5] and in references therein). Kada et al. [2] defined the concept of $w$-distance in a metric space as follows.

Definition 1.1 (see [2]). Let $X$ be a metric space endowed with a metric $d$. A function $p: X \times X \rightarrow[0, \infty)$ is called a $w$-distance on $X$ if it satisfies the following properties:

(1) $p(x, z) \leq p(x, y)+p(y, z)$ for any $x, y, z \in X$,

(2) $p$ is lower semicontinuous in its second variable, that is, if $x \in X$ and $y_{n} \rightarrow y$ in $X$ then $p(x, y) \leq \liminf _{n \rightarrow \infty} p\left(x, y_{n}\right)$,

(3) for each $\epsilon>0$, there exists $\delta>0$ such that $p(z, x) \leq \delta$ and $p(z, y) \leq \delta$ imply $d(x, y) \leq \epsilon$. 
We denote by $\Phi$ the set of functions $\varphi:[0,+\infty) \rightarrow[0,+\infty)$ satisfying the following hypotheses:

(c1) $\varphi$ is continuous and nondecreasing,

(c2) $\varphi(t)=0$ if and only if $t=0$. hypotheses:

We denote by $\Psi$ the set of functions $\psi:[0,+\infty) \rightarrow[0,+\infty)$ satisfying the following

(h1) $\psi$ is right continuous and nondecreasing,

(h2) $\psi(t)<t$ for all $t>0$.

Let $p$ be a $w$-distance on metric space $(X, d), \varphi \in \Phi$ and $\psi \in \Psi$. A map $T$ from $X$ into itself is a $(\varphi, \psi, p)$-contractive map on $X$ if for each $x, y \in X, \varphi p(T x, T y) \leq \psi \varphi p(x, y)$.

The following lemmas are used in the next section.

Lemma 1.2 (see [3]). If $\psi \in \Psi$, then $\lim _{n \rightarrow \infty} \psi^{n}(t)=0$ for each $t>0$, and if $\varphi \in \Phi,\left\{a_{n}\right\} \subseteq[0, \infty)$ and $\lim _{n \rightarrow \infty} \varphi\left(a_{n}\right)=0$, then $\lim _{n \rightarrow \infty} a_{n}=0$.

Lemma 1.3 (see [2]). Let $(X, d)$ be a metric space and let $p$ be a w-distance on $X$.

(i) If $\left\{x_{n}\right\}$ is a sequence in $X$ such that $\lim _{n} p\left(x_{n}, x\right)=\lim _{n} p\left(x_{n}, y\right)=0$, then $x=y$. In particular, if $p(z, x)=p(z, y)=0$, then $x=y$.

(ii) If $p\left(x_{n}, y_{n}\right) \leq \alpha_{n} p\left(x_{n}, y\right) \leq \beta_{n}$ for any $n \in \mathbb{N}$, where $\left\{\alpha_{n}\right\}$ and $\left\{\beta_{n}\right\}$ are sequences in $[0, \infty)$ converging to 0 , then $\left\{y_{n}\right\}$ converges to $y$.

(iii) Let $p$ be a w-distance on metric space $(X, d)$ and $\left\{x_{n}\right\}$ a sequence in $X$ such that for each $\varepsilon>0$ there exist $N_{\varepsilon} \in N$ such that $m>n>N_{\varepsilon}$ implies $p\left(x_{n}, x_{m}\right)<\varepsilon$ (or $\left.\lim _{m, n \rightarrow \infty} p\left(x_{n}, x_{m}\right)=0\right)$, then $\left\{x_{n}\right\}$ is a Cauchy sequence.

Note that if $p(a, b)=p(b, a)=0$ and $p(a, a) \leq p(a, b)+p(b, a)=0$, then $p(a, a)=0$ and, by Lemma 1.3, $a=b$.

In [3], Razani et al. proved a fixed point theorem for $(\varphi, \psi, p)$-contractive mappings, which is a new version of the main theorem in [1], by considering the concept of the wdistance.

The main aim of this paper is to present some generalization fixed point Theorems by Kada et al. [2], Hicks and Rhoades [6] and several other results with respect to $(\varphi, \psi, p)$ contractive maps on a complete metric space.

\section{2. $(\varphi, \psi, p)$-Contractive Maps}

In the next theorem we state one of the main results of this paper generalizing Theorem 4 of [2]. In what follows, we use $\varphi p$ to denote the composition of $\varphi$ with $p$.

Theorem 2.1. Let $p$ be a $w$-distance on complete metric space $(X, d), \varphi \in \Phi$ and $\psi \in \Psi$. Suppose $T: X \rightarrow X$ is a map that satisfies

$$
\varphi p\left(T x, T^{2} x\right) \leq \psi(\varphi p(x, T x))
$$


for each $x \in X$ and that

$$
\inf \{p(x, y)+p(x, T x): x \in X\}>0
$$

for every $y \in X$ with $y \neq T y$. Then there exists $u \in X$ such that $u=T u$. Moreover, if $v=T v$, then $p(v, v)=0$.

Proof. Fix $x \in X$. Set $x_{n+1}=T x_{n}$ with $x_{0}=x$. Then by (2.1)

$$
\begin{aligned}
\varphi p\left(x_{n}, x_{n+1}\right) & \leq \psi \varphi p\left(x_{n-1}, x_{n}\right) \\
& \leq \psi^{2} \varphi p\left(x_{n-2}, x_{n-1}\right) \\
& \leq \cdots \leq \psi^{n}\left(\varphi p\left(x_{0}, x_{1}\right)\right)
\end{aligned}
$$

thus $\lim _{n} \varphi p\left(x_{n}, x_{n+1}\right)=0$ and Lemma 1.2 implies

$$
\lim _{n \rightarrow \infty} p\left(x_{n}, x_{n+1}\right)=0
$$

and similarly

$$
\lim _{n \rightarrow \infty} p\left(x_{n+1}, x_{n}\right)=0
$$

Now we proof that $\left\{x_{n}\right\}$ is a Cauchy sequence. By triangle inequality, continuity of $\varphi$ and (2.4), we have

$$
\varphi p\left(x_{n}, x_{n+2}\right) \leq \psi \varphi\left[p\left(x_{n}, x_{n+1}\right)+p\left(x_{n+1}, x_{n+2}\right)\right] \longrightarrow 0,
$$

as $n \rightarrow \infty$ and so $\lim _{n \rightarrow \infty} \varphi p\left(x_{n}, x_{n+2}\right)=0$ which concludes

$$
\lim _{n \rightarrow \infty} p\left(x_{n}, x_{n+2}\right)=0
$$

By induction, for any $k>0$ we have

$$
\lim _{n \rightarrow \infty} p\left(x_{n}, x_{n+k}\right)=0
$$

So, by Lemma $1.3,\left\{x_{n}\right\}$ is a Cauchy sequence, and since $X$ is complete, there exists $u \in X$ such that $x_{n} \rightarrow u$ in $X$.

Now we prove that $u$ is a fixed point of $T$.

From (2.8), for each $\varepsilon>0$, there exists $N_{\varepsilon} \in \mathbb{N}$ such that $n>N_{\varepsilon}$ implies $p\left(x_{N_{\varepsilon}}, x_{n}\right)<\varepsilon$ but $x_{n} \rightarrow u$ and $p(x, \cdot)$ is lower semicontinuous, thus

$$
p\left(x_{N_{\varepsilon}}, u\right) \leq \liminf _{n \rightarrow \infty} p\left(x_{N_{\varepsilon}}, x_{n}\right) \leq \varepsilon .
$$


Therefore, $p\left(x_{N_{\varepsilon}}, u\right) \leq \varepsilon$. Set $\varepsilon=1 / k, N_{\varepsilon}=n_{k}$ and we have

$$
\lim _{k \rightarrow \infty} p\left(x_{n_{k}}, u\right)=0
$$

Now, assume that $u \neq T u$. Then by hypothesis, we have

$$
0<\inf \{p(x, u)+p(x, T x): x \in X\} \leq \inf \left\{p\left(x_{n}, u\right)+p\left(x_{n}, x_{n+1}\right): n \in \mathbb{N}\right\} \rightarrow 0
$$

as $n \rightarrow \infty$ by (2.4) and (2.10). This is a contradiction. Hence $u=T u$.

If $v=T v$, we have

$$
\varphi p(v, v)=\varphi p\left(T v, T^{2} v\right) \leq \psi \varphi p(v, T v)=\psi \varphi p(v, v)<\varphi p(v, v) .
$$

This is a contradiction. So $\varphi p(v, v)=0$, and by hypothesis $p(v, v)=0$.

Here we give a simple example illustrating Theorem 2.1. In this example, we will show that Theorem 4 in [2] cannot be applied.

Example 2.2. Let $X=\{(1 / n) \mid n \in \mathbb{N}\} \cup\{0\}$, which is a complete metric space with usual metric $d$ of reals. Moreover, by defining $p(x, y)=y, p$ is a $w$-distance on $(X, d)$. Let $T: X \rightarrow X$ be a map as $T(1 / n)=1 /(n+1), T 0=0$. Suppose $\varphi(t)=t^{1 / t}$ is a continuous and strictly nondecreasing map and $\psi(t)=(1 / 3) t$, for any $t>0$. We have

$$
\sup _{x \in X} \frac{p\left(T x, T^{2} x\right)}{p(x, T x)}=1,
$$

and so there is not any $r \in[0,1)$ such that $p\left(T x, T^{2} x\right) \leq r p(x, T x)$, and hence Theorem 4 in [2] dose not work. But

$$
\begin{aligned}
\varphi p\left(T x, T^{2} x\right) & =p\left(T x, T^{2} x\right)^{1 / p\left(T x, T^{2} x\right)}=\left(\frac{1}{n+2}\right)^{n+2} \leq \frac{1}{3}\left(\frac{1}{n+1}\right)^{n+1} \\
& =\frac{1}{3} p(x, T x)^{1 / p(x, T x)}=\psi \varphi p(x, T x)
\end{aligned}
$$

because for any $n \in \mathbb{N}$ we have $((n+1) /(n+2))^{n+1} 1 /(n+2) \leq 1 / 3$. Also for any $n \in \mathbb{N}$ we have $1 / n \neq T(1 / n)$. So for arbitrary $n \in \mathbb{N}, \inf \{p(1 / m, 1 / n)+p(1 / m, 1 /(m+1)): m \in \mathbb{N}\}=1 / n>0$, hence $T$ is satisfied in Theorem 2.1. We note that 0 is a fixed point for $T$.

The next examples show the role of the conditions (2.1) and (2.2).

Example 2.3. Let $X=[-1,1], d(x, y)=|x-y|$, and define $p: X \rightarrow X$ by $p(x, y)=|3 x-3 y|$, where $x, y \in X$. Set $\psi(t)=r t$ and $\varphi(t)=t$ for all $t \in[0, \infty)$. Let us define $T: X \rightarrow X$ by $T 0=1$ and $T x=x / 10$ if $x \neq 0$. We have

$$
\varphi p\left(T 0, T^{2} 0\right)=p\left(T 0, T^{2} 0\right)=p\left(1, \frac{1}{10}\right)=3-\frac{3}{10} \leq 3=\frac{1}{3} p(0, T 0)=\psi \varphi p(0, T 0) .
$$




$$
\begin{aligned}
& \text { If } x \neq 0 \text {, then } \\
& \varphi p\left(T x, T^{2} x\right)=p\left(T x, T^{2} x\right)=p\left(\frac{x}{10}, \frac{x}{100}\right)=\frac{1}{10}\left|3 x-\frac{3 x}{10}\right| \leq \frac{1}{3} p(x, T x)=\psi \varphi p(x, T x)
\end{aligned}
$$

and hence (2.1) holds.

Now, we remark that $0 \neq T(0)$, and

$$
\inf _{n \in \mathbb{N}} p\left(T^{n}(x), 0\right)+p\left(T^{n}(x), T T^{n}(x)\right)=0 \quad \text { for every } x \in \mathrm{X}
$$

Thus, the condition (2.2) is not satisfied, and there is no $z \in X$ with $T z=z$. In this case we observe that Theorem 2.1 is invalid without condition (2.2).

Example 2.4. Let $X=[2, \infty) \cup\{0,1\}, d(x, y)=|x-y|, x, y \in X$, and set $p=d$. Let $\psi, \varphi$ be as Example 2.3. Let us define $T: X \rightarrow X$ by $T 0=1$ and $T x=0$ if $x \neq 0$. Clearly, $T$ has no fixed point in $X$. Now, for each $x \in X$ and that

$$
\inf \{d(x, y)+d(x, T x): x \in X\}>0
$$

for every $y \in X$ with $y \neq T y$, so condition (2.2) is satisfied. But, for $x=0, d\left(T x, T^{2} x\right)>$ $r d(x, T x)$ for any $r \in[0,1)$. Hence, condition (2.1) dose not hold. We note that Theorem 2.1 dose not work without condition (2.1).

Suppose $\theta: \mathbb{R}^{+} \rightarrow \mathbb{R}^{+}$is Lebesgue-integrable mapping which is summable and $\int_{0}^{\varepsilon} \theta(\eta) d \eta>0$, for each $\varepsilon>0$. Now, in the next corollary, set $\varphi(t)=\int_{0}^{t} \theta(\eta) d \eta$ and $\psi(t)=c t$, where $c \in[0,1[$. Then, $\varphi \in \Phi$ and $\psi \in \Psi$. Hence we can conclude the following corollary as a special case.

Corollary 2.5. Let $T$ be a selfmap of a complete metric space $(X, d)$ satisfying

$$
\int_{0}^{d\left(T x, T^{2} x\right)} \theta(t) d t \leq c \int_{0}^{d(x, T x)} \theta(t) d t
$$

for all $x \in X$. Suppose that

$$
\inf \{d(x, y)+d(x, T x): x \in X\}>0 \text { for every } y \in X
$$

with $y \neq T y$. Then there exists $a u \in X$ such that $T u=u$.

Note that Corollary 2.5 is invalid without condition (2.20). For example, take $X=$ $\{0\} \cup\left\{1 / 2^{n}: n \geq 1\right\}$, which is a complete metric space with usual metric $d$ of reals. Define $T: X \rightarrow X$ by $T(0)=1 / 2$ and $T\left(1 / 2^{n}\right)=1 / 2^{n-1}$ for $n \geq 1$. Set $\varphi(t) \equiv 1$. It is easy to check that $\int_{0}^{d\left(T x, T^{2} x\right)} \varphi(t) d t \leq(1 / 2) \int_{0}^{d(x, T x)} \varphi(t) d t$, for any $x \in X$; however, $y \neq T y$ for any $y \in X$ and $\inf \{d(x, y)+d(x, T x): x \in X\}=0$. Clearly, $T$ has got no fixed point in $X$. 
Remark 2.6. From Theorem 2.1, we can obtain Theorem 4 in [2] as a special case. For this, in the hypotheses of Theorem 2.1, set $\psi(t)=r t$ and $\varphi(t)=t$ for all $t \in[0, \infty)$.

Corollary 2.7. Let $p$ be a $w$-distance on complete metric space $(X, d), \varphi \in \Phi$ and $\psi \in \Psi$. Suppose $T$ is a continuous mapping for $X$ into itself such that (2.1), is satisfied. Then there exists $u \in X$ such that $u=T u$. Moreover, if $v=T v$, then $p(v, v)=0$.

Proof. Assume that there exists $y \in X$ with $y \neq T y$ and $\inf \{p(x, y)+p(x, T x): x \in X\}=0$. Then there exists a sequence $\left\{x_{n}\right\}$ such that

$$
p\left(x_{n}, y\right)+p\left(x_{n}, T x_{n}\right) \longrightarrow 0
$$

as $n \rightarrow \infty$. Hence $p\left(x_{n}, y\right) \rightarrow 0$ and $p\left(x_{n}, T x_{n}\right) \rightarrow 0$ as $n \rightarrow \infty$. Lemma 1.3 implies that $T x_{n} \rightarrow y$ as $n \rightarrow \infty$. Now by assumption

$$
\varphi p\left(T x_{n}, T^{2} x_{n}\right) \leq \psi\left(\varphi p\left(x_{n}, T x_{n}\right)\right)
$$

and so $\varphi p\left(T x_{n}, T^{2} x_{n}\right) \rightarrow 0$ as $n \rightarrow \infty$. By Lemma $1.2, p\left(T x_{n}, T^{2} x_{n}\right) \rightarrow 0$ as $n \rightarrow \infty$. We also have

$$
p\left(x_{n}, T^{2} x_{n}\right) \leq p\left(x_{n}, T x_{n}\right)+p\left(T x_{n}, T^{2} x_{n}\right)
$$

hence $p\left(x_{n}, T^{2} x_{n}\right) \rightarrow 0$ as $n \rightarrow \infty$. By Lemma 1.3, we conclude that $\left\{T^{2} x_{n}\right\}$ converges to $y$. Since $T$ is continuous, we have

$$
T y=T\left(\lim _{n \rightarrow \infty} T x_{n}\right)=\lim _{n \rightarrow \infty} T^{2} x_{n}=y
$$

This is a contradiction. Therefore, if $y \neq T y$, then $\inf \{p(x, y)+p(x, T x): x \in X\}>0$. So, Theorem 2.1 gives desired result.

In Example 2.3, $T$ is satisfied in condition (2.1), but it is not continuous. So, the hypotheses in Corollary 2.7 are not satisfied. We note that $T$ has no fixed point.

It is an obvious fact that, if $f: X \rightarrow X$ is a map which has a fixed point $x \in X$, then $x$ is also a fixed point of $f^{n}$ for every natural number $n$. However, the converse is false. If a map satisfies $F(f)=F\left(f^{n}\right)$ for each $n \in \mathbb{N}$, where $F(f)$ denotes a set of all fixed points of $f$, then it is said to have property $P[7,8]$. The following theorem extends and improves Theorem 2 of [7].

Theorem 2.8. Let $(X, d)$ be a complete metric space with w-distance $p$ on $X$. Suppose $T: X \rightarrow X$ satisfies

(i)

$$
\varphi p\left(T x, T^{2} x\right) \leq \psi \varphi p(x, T x), \quad \forall x \in X
$$

or

(ii) with strict inequality, $\psi \equiv 1$ and for all $x \in X, x \neq T x$. If $F(T) \neq \emptyset$, then $T$ has property $P$. 
Proof. We shall always assume that $n>1$, since the statement for $n=1$ is trivial. Let $u \in F\left(T^{n}\right)$. Suppose that $T$ satisfies (i). Then,

$$
\varphi p(u, T u)=\varphi p\left(T^{n} u, T T^{n} u\right) \leq \varphi \varphi p\left(T^{n-1} u, T T^{n-1} u\right) \leq \cdots \leq \psi^{n} \varphi p(u, T u)
$$

and so $p(u, T u)=0$. Now from

$$
\varphi p(u, u)=\psi \varphi p\left(u, T^{n} u\right) \leq \sum_{i=0}^{n-1} \psi \varphi p\left(T^{i} u, T^{i+1} u\right)=0,
$$

we have $p(u, u)=0$. Hence, by Lemma 1.3, we have $u=T u$, and $u \in F(T)$. Suppose that $T$ satisfies (ii). If $T u=u$, then there is nothing to prove. Suppose, if possible, that $T u \neq u$. Then a repetition of the argument for case (i) leads to $\varphi p(u, T u)<\psi \varphi p(u, T u)$, that is a contradiction. Therefore, in all cases, $u=T u$ and $F\left(T^{n}\right)=F(T)$.

The following theorem extends Theorem 2.1 of [6]. A function $G$ mapping $X$ into the real is $T$-orbitally lower semicontinuous at $z$ if $\left\{x_{n}\right\}$ is a sequence in $O(x, \infty)$ and $x_{n} \rightarrow z$ implies that $G(p) \leq \liminf _{n \rightarrow \infty} G\left(x_{n}\right)$.

Theorem 2.9. Let $(X, d)$ be a complete metric space with $w$-distance $p$ on $X$. Suppose $T: X \rightarrow X$ and there exists an $x$ such that

$$
\varphi p\left(T y, T^{2} y\right) \leq \psi \varphi p(y, T y), \quad \forall y \in O(x, \infty) .
$$

Then,

(i) $\lim T^{n} x=z$ exists,

(ii)

$$
\varphi p\left(T^{n} x, z\right) \leq \frac{\psi^{n}}{1-\psi} \varphi p(x, T x) \quad \text { for } n \geq 1
$$

(iii) $p(z, T z)=0$ if and only if $G(x)=p(x, T x)$ is T-orbitally lower semicontinuous at $z$.

Proof. Observe that (i) and (ii) are immediate from the proof of Theorem 2.1. We prove (iii). It is clear that $p(z, T z)=0$ impling $G(x)$ is $T$-orbitally lower semicontinuous at $z$. $x_{n}=T^{n} x \rightarrow z$ and $G$ is $T$-orbitally lower semicontinuous at $x$ implies

$$
0 \leq \varphi p(z, T z)=\varphi G(z) \leq \liminf _{n \rightarrow \infty} \varphi G\left(x_{n}\right)=\liminf _{n \rightarrow \infty} \psi \varphi p\left(x_{n}, T x_{n}\right) \leq \liminf _{n \rightarrow \infty} \psi^{n} \varphi p(x, T x)=0 .
$$

So, $p(z, T z)=0$.

The mapping $T$ is orbitally lower semicontinuous at $u \in X$ if $\lim _{k \rightarrow \infty} T^{n_{k}} x=u$ implies that $\lim _{k \rightarrow \infty} T^{n_{k}+1} x=T u$. In the following, we improve Theorem 2 of [9] that it is correct form Theorem 1 of [7]. 
Theorem 2.10. Let $p$ be a $w$-distance on complete metric space $(X, d), \varphi \in \Phi$ and $\psi \in \Psi$. Suppose $T: X \rightarrow X$ is orbitally lower semicontinuous map on $X$ that satisfies

$$
\varphi p\left(T x, T^{2} x\right) \leq \psi(\varphi p(x, T x))
$$

for each $x \in X$. Then there exists $u \in X$ such that $u \in F(T)$. Moreover, if $v=T v$, then $p(v, v)=0$.

Proof. Observe that the sequence $\left\{x_{n}\right\}$ is a Cauchy sequence immediate from the proof of Theorem 2.1 and so there exists a point $u$ in $X$ such that $x_{n} \rightarrow u$ as $n \rightarrow \infty$. Since $T$ is orbitally lower semicontinuous at $u$, we have $p(u, T u) \leq \liminf _{n \rightarrow \infty} p\left(x_{n}, x_{n+1}\right)=0$. Now, we have

$$
\varphi p(u, T u) \leq \varphi \liminf _{n \rightarrow \infty} p\left(x_{n}, x_{n+1}\right)=\varphi(0)=0,
$$

and so $p(u, T u)=0$. Similarly, $p(T u, u)=0$. Hence, $u \in F(T)$. By Theorem 2.1 we can conclude that if $v=T v$, then $p(v, v)=0$.

The following example shows that Theorem 2 in [9] cannot be applicable. So our generalization is useful.

Example 2.11. Let $=[0, \infty)$ be a metric space with metric $d$ defined by $d(x, y)=(40 / 3) \mid x-$ $y \mid, x, y \in X$, which is complete. We define $p: X \rightarrow X$ by $p(x, y)=(1 / 3)|y|$. Let $\varphi$ be as defined before in Corollary 2.5 and $\psi(t)=(1 / 10) t, t>0$. Assume that $T: X \rightarrow X$ by $T x=x / 10$ for any $x \in X$. We have, $d\left(T x, T^{2} x\right)=(4 / 3) d(x, T x), x \in X$, and so Theorem 2 in [9] dose not work. But

$$
\varphi p\left(T x, T^{2} x\right) \leq \psi(\varphi p(x, T x))
$$

for each $x \in X$. Hence by Theorem 2.10 there exists a fixed point for $T$. We note that 0 is fixed point for $T$.

\section{3. $(\varphi, k, p)$-Contractive Maps}

In this section we obtain fixed points for $(\varphi, k, p)$-contractive maps (i.e., $(\varphi, \psi, p)$-contractive maps that $\psi(t)=k$ for all $t \in[0, \infty)$, where $k \in[0,1))$.

In 1969, Kannan [10] proved the following fixed point theorem. Contractions are always continuous and Kannan maps are not necessarily continuous.

Theorem 3.1 (see $[10])$. Let $(X, d)$ be a complete metric space. Let $T$ be a Kannan mapping on $X$, that is, there exists $k \in[0,1 / 2)$ such that

$$
d(T x, T y) \leq k(d(x, T x)+d(y, T y))
$$

for all $x, y \in X$. Then, $T$ has a unique fixed point in $X$. For each $x \in X$, the iterative sequence $\left\{T^{n} x\right\}_{n \geq 1}$ converges to the fixed point. 
In the next theorem, we generalize this theorem as follows.

Theorem 3.2. Let $(X, d)$ be a complete metric space. Let $T$ be a $(\varphi, k)$-Kannan mapping on $X$, that is, there exists $k \in[0,1 / 2)$ such that

$$
\varphi d(T x, T y) \leq k(\varphi d(x, T x)+\varphi d(y, T y))
$$

for all $x, y \in X$. Then, $T$ has a unique fixed point in $X$. For each $x \in X$, the iterative sequence $\left\{T^{n} x\right\}_{n \geq 1}$ converges to the fixed point.

Proof. Let $x \in X$ and define $x_{n+1}=T^{n} x$ for any $n \in N$, and set $r=k /(1-k)$. Then, $r \in[0,1)$,

$$
\varphi d\left(T x, T^{2} x\right) \leq k\left(\varphi d(x, T x)+\varphi d\left(T x, T^{2} x\right)\right)
$$

and so

$$
\varphi d\left(T x, T^{2} x\right) \leq r \varphi d(x, T x)
$$

Then, from the proof of Theorem 2.1, $\lim T^{n} x=z$ exists. From (3.4), we have

$$
\varphi d\left(T^{n} x, T z\right) \leq r \varphi d\left(T^{n-1} x, z\right) \leq \frac{r^{n}}{1-r} \varphi d(x, T x) \text { for } n \geq 1
$$

Thus, $\lim T^{n} x=T z$, and so $z=T z$. Clearly, $z$ is unique. This completes the proof.

The set of all subadditive functions $\varphi$ in $\Phi$ is denoted by $\Phi^{\prime}$. In the following theorems, we generalize Theorems 3.4 and 3.5 due to Suzuki and Takahashi [4].

Theorem 3.3. Let $p$ be a $w$-distance on complete metric space $(X, d), \varphi \in \Phi^{\prime}$ and $T$ be a selfmap. Suppose there exists $k \in[0,1 / 2)$ such that

(i) $\varphi p\left(T x, T^{2} x\right) \leq k \varphi p\left(x, T^{2} x\right)$ for each $x \in X$,

(ii) $\inf \{p(x, z)+p(x, T x): x \in X\}>0$ for every $z \in X$ with $z \neq T z$.

Then $T$ has a fixed point in X. Moreover, if $v$ is a fixed point of $T$, then $p(v, v)=0$.

Proof. Fix $x \in X$. Define $x_{0}=x$ and $x_{n}=T^{n} x_{0}$ for every $n \in \mathbb{N}$. Put $r=k /(1-k)$. Then, $0 \leq r<1$. By hypothesis, since $\varphi \in \Phi^{\prime}$, we have

$$
\varphi p\left(x_{n}, x_{n+1}\right) \leq k \varphi p\left(x_{n-1}, x_{n+1}\right) \leq k \varphi p\left(x_{n-1}, x_{n}\right)+k \varphi p\left(x_{n}, x_{n+1}\right),
$$

for all $n \in \mathbb{N}$. It follows that

$$
\varphi p\left(x_{n}, x_{n+1}\right) \leq r \varphi p\left(x_{n-1}, x_{n}\right) \leq \cdots \leq r^{n} \varphi p\left(x_{0}, x_{1}\right),
$$


for all $n \in \mathbb{N}$. Using the similar argument as in the proof of Theorem 2.1, we can prove that the sequence $\left\{u_{n}\right\}$ is Cauchy and so there exists $u \in X$ such that $x_{n} \rightarrow u$ as $n \rightarrow \infty$. Also, we have $u \in F(T)$. Since

$$
\varphi p(v, v)=\varphi p\left(T v, T^{2} v\right) \leq k \varphi p\left(v, T^{2} v\right)=k \varphi p(v, v)
$$

we have $\varphi p(v, v)=0$ and so $p(v, v)=0$. The proof is completed.

Corollary 3.4. Let $p$ be a $w$-distance on complete metric space $(X, d), \varphi \in \Phi^{\prime}$ and let $T$ be a continuous map. Suppose there exists $k \in[0,1 / 2)$ such that

$$
\varphi p\left(T x, T^{2} x\right) \leq k \varphi p\left(x, T^{2} x\right)
$$

for each $x \in X$.

Then $T$ has a fixed point in $X$. Moreover, if $v$ is a fixed point of $T$, then $p(v, v)=0$.

Proof. It suffices to show that $\inf \{p(x, z)+p(x, T x): x \in X\}>0$ for every $u \in X$ with $u \neq T u$. Assume that there exists $u \in X$ with $u \neq T u$ and $\inf \{p(x, u)+p(x, T x): x \in X\}=0$. Then there exists a sequence $\left\{x_{n}\right\}$ in $X$ such that $\lim _{n \rightarrow \infty}\left[p\left(x_{n}, u\right)+p\left(x_{n}, T x_{n}\right)\right]=0$. It follows that $p\left(x_{n}, u\right) \rightarrow 0$ and $p\left(x_{n}, T x_{n}\right) \rightarrow 0$ as $n \rightarrow \infty$. Hence, $T x_{n} \rightarrow u$. On the other hand, since $\varphi \in \Phi^{\prime}$ and (3.9), we have

$$
\varphi p\left(x_{n}, T^{2} x_{n}\right) \leq \varphi p\left(x_{n}, T x_{n}\right)+\varphi p\left(T x_{n}, T^{2} x_{n}\right) \leq \varphi p\left(x_{n}, T x_{n}\right)+k \varphi p\left(x_{n}, T^{2} x_{n}\right),
$$

and hence

$$
\varphi p\left(x_{n}, T^{2} x_{n}\right) \leq \frac{1}{1-k} \varphi p\left(x_{n}, T x_{n}\right)
$$

for all $n \in \mathbb{N}$. Thus, $p\left(x_{n}, T^{2} x_{n}\right) \rightarrow 0$ as $n \rightarrow \infty$. Therefore, $T^{2} x_{n} \rightarrow u$. Since $T: X \rightarrow X$ is continuous, we have

$$
T(u)=T\left(\lim _{n \rightarrow \infty} T x_{n}\right)=\lim _{n \rightarrow \infty} T^{2} x_{n}=u,
$$

which is a contradiction. Therefore, using Theorem 3.3, $p(v, v)=0$. This completes the proof.

Question 1. Can we generalize Theorems 3.2, 3.3, and Corollary 3.4 for $(\varphi, \psi, p)$-contractive maps?

\section{References}

[1] A. Branciari, "A fixed point theorem for mappings satisfying a general contractive condition of integral type," International Journal of Mathematics and Mathematical Sciences, vol. 29, no. 9, pp. 531$536,2002$. 
[2] O. Kada, T. Suzuki, and W. Takahashi, "Nonconvex minimization theorems and fixed point theorems in complete metric spaces," Mathematica Japonica, vol. 44, no. 2, pp. 381-391, 1996.

[3] A. Razani, Z. Mazlumi Nezhad, and M. Boujary, "A fixed point theorem for w-distance," Applied Sciences, vol. 11, pp. 114-117, 2009.

[4] T. Suzuki and W. Takahashi, "Fixed point theorems and characterizations of metric completeness," Topological Methods in Nonlinear Analysis, vol. 8, no. 2, pp. 371-382, 1996.

[5] W.-S. Du, "Fixed point theorems for generalized Hausdorff metrics," International Mathematical Forum, vol. 3, no. 21-24, pp. 1011-1022, 2008.

[6] T. L. Hicks and B. E. Rhoades, "A Banach type fixed-point theorem," Mathematica Japonica, vol. 24, no. 3, pp. 327-330, 1979/80.

[7] B. E. Rhoades and M. Abbas, "Maps satisfying generalized contractive conditions of integral type for which $F(T)=F\left(T^{n}\right)$," International Journal of Pure and Applied Mathematics, vol. 45, no. 2, pp. 225-231, 2008.

[8] G. S. Jeong and B. E. Rhoades, "Maps for which $F(T)=F\left(T^{n}\right)$," Fixed Point Theory and Applications, vol. 6, pp. 87-131, 2005.

[9] H. Lakzian and B. E. Rhoades, "Maps satisfying generalized contractive contractions of integral type for which $F(T)=F\left(T^{n}\right)$," submitted to International Journal of Pure and Applied Mathematical Sciences.

[10] R. Kannan, "Some results on fixed points. II," The American Mathematical Monthly, vol. 76, pp. 405-408, 1969. 




Advances in

Operations Research

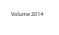



\section{The Scientific} World Journal
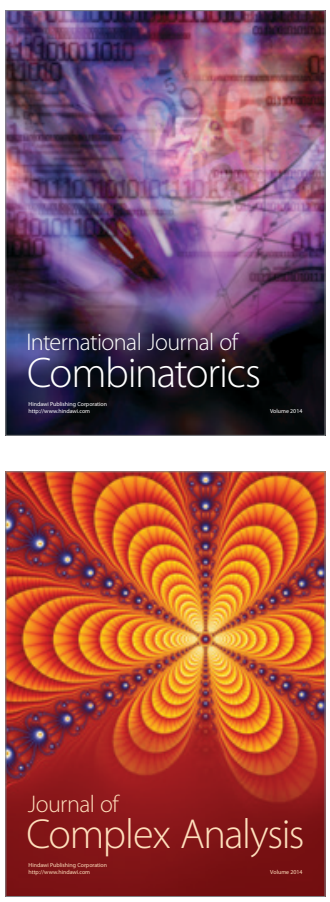

International Journal of

Mathematics and

Mathematical

Sciences
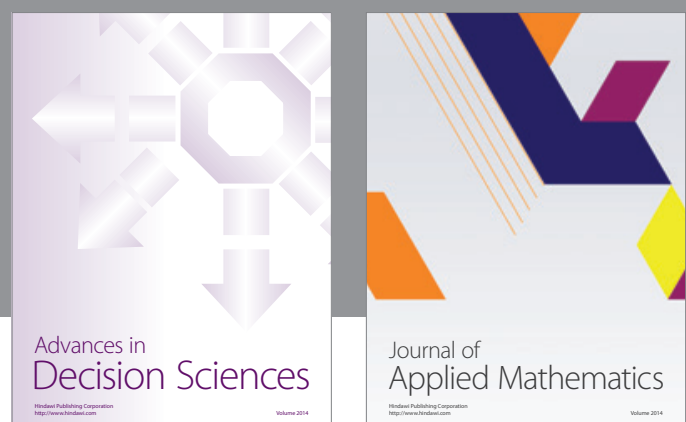

Journal of

Applied Mathematics
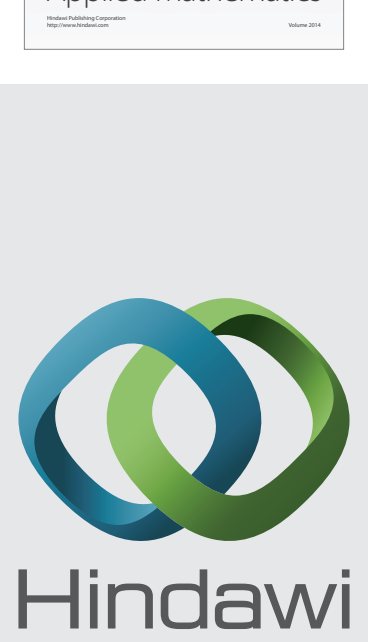

Submit your manuscripts at http://www.hindawi.com
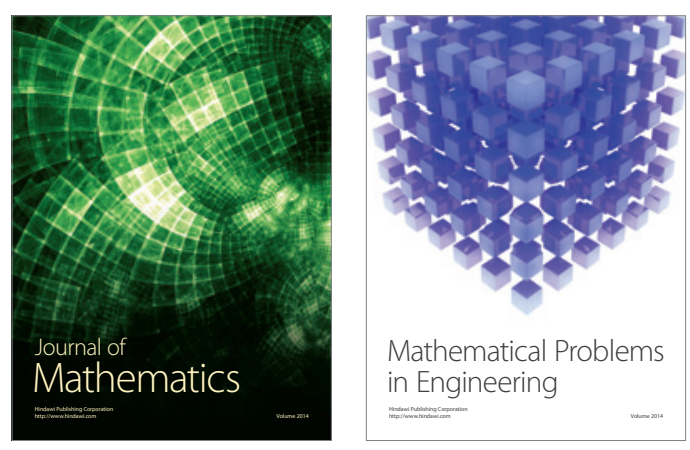

Mathematical Problems in Engineering
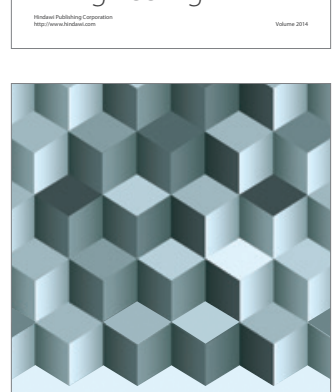

Journal of

Function Spaces
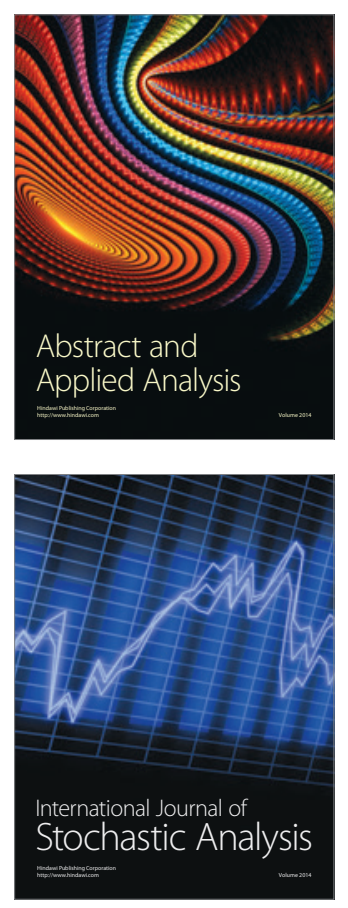

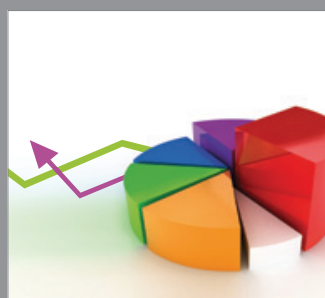

ournal of

Probability and Statistics

Promensencen
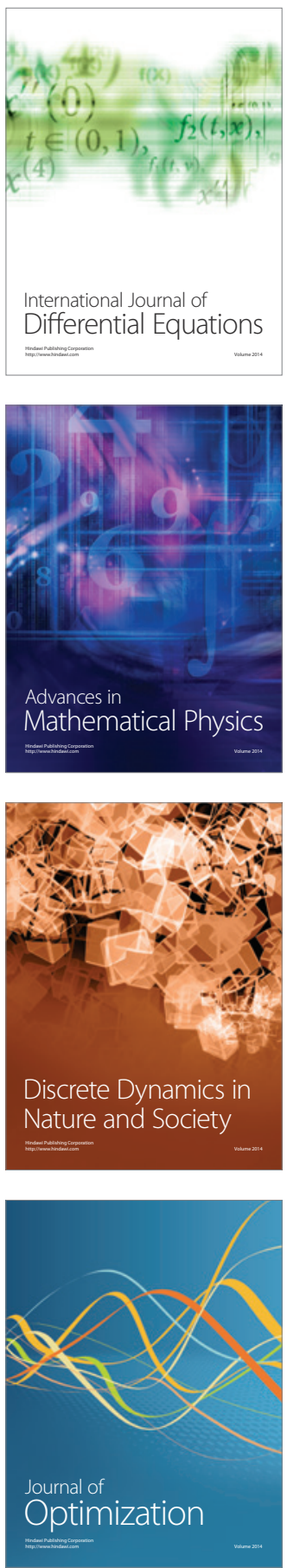